\title{
Operaciones portuarias de petróleo crudo: Una revisión bibliográfica sobre simulación e investigación operativa
}

\author{
Port operations on crude oil: \\ A bibliographic review on simulation and operations research
}

Ignacio de la Peña Zarzuelo ${ }^{1} \quad$ María Jesús Freire Seoane ${ }^{2} \quad$ Beatriz López Bermúdez ${ }^{2 *}$

Recibido 16 de mayo de 2019, aceptado 10 de febrero de 2021

Received: May 16, 2019 Accepted: February 10, 2021

\begin{abstract}
RESUMEN
Actualmente, el tráfico marítimo de crudo de petróleo y sus derivados sigue siendo un tráfico relevante, tanto desde un punto de vista geoestratégico como a nivel de volumen transportado. Dado el desequilibrio regional en términos de reservas, producción y consumo, se genera, por tanto, un importante tráfico marítimo, pero también una necesidad de la industria de optimizar los procesos con el objetivo de minimizar los costes y dar una respuesta eficiente en términos logísticos.

Desde esta perspectiva, las técnicas de investigación operativa y la modelización de los tráficos para su optimización se presentan como una metodología muy conveniente y, a lo largo de los últimos setenta años, han empezado a publicarse estudios científicos con diferentes enfoques cualitativos y cuantitativos. El objetivo de esta investigación es recopilar, analizar y clasificar los modelos de tráfico de crudo de petróleo y sus derivados y ordenar la temática de las innovaciones introducidas. Para alcanzar este objetivo se realiza un recorrido bibliográfico conceptual indicando como se han ido introduciendo las sucesivas mejoras y, al mismo tiempo, eliminando las restricciones iniciales.
\end{abstract}

Palabras clave: Inventory routing problem (IRP); lightering optimization problem (LOP); cargo scheduling with blending problem (CSBP); pricing, inventory \& scheduling combined problem (PISCP); routing and scheduling problem (RSP).

\begin{abstract}
The maritime traffic of crude oil and derivatives is still very relevant, both from a geostrategic perspective and regarding the total volume of liquid bulk shipped. Due to the regional imbalance in terms of reserves, production and consumption, important maritime traffic is generated, and a necessity for the industry of optimizing these processes, with the goal to minimize costs and give an efficient answer in logistic terms. From this perspective, the operation research techniques, and the traffic modeling techniques aimed at optimization, can be presented as a very convenient methodology over the last seventy years, a stream of scientific publications has emerged with different quantitative and qualitative focus.

This research aims to gather, analyze and classify the traffic models of crude oil and derivatives and to order the innovation concepts gradually introduced. In order to achieve this goal, conceptual bibliographic research is performed showing how the subsequent improvements have been introduced and, at the same time, erasing the initial restrictions.
\end{abstract}

Keywords: Inventory routing problem (IRP); lightering optimization problem (LOP); cargo scheduling with blending problem (CSBP); pricing, inventory \& scheduling combined problem (PISCP); routing and scheduling problem (RSP).

\footnotetext{
1 Universidad Politécnica de Madrid. Departamento de Ingeniería Civil: Construcción. Madrid, España. E-mail: i.delapena@upm.es

2 Universidad de A Coruña. Facultad de Economía y Empresa. A Coruña, España. E-mail: maje@udc.es; beatriz.lopez2@udc.es

* Autor de correspondencia: beatriz.lopez2@udc.es
} 


\section{INTRODUCCIÓN}

El petróleo sigue siendo la principal fuente de generación de energía primaria en el mundo con más de 3,501 millones de toneladas de crudo y productos importadas en 2018 lo que supone un $32 \%$ del total del consumo energético mundial [16]. Sin embargo, las reservas, producción y consumo de petróleo están desigualmente distribuidas geográficamente lo que lleva a la necesidad de transportar grandes volúmenes a largas distancias.

En la Tabla 1 se observa que el $48 \%$ de las reservas en 2018 estaban localizadas en el Medio Este (Arabia Saudí, Irán, Irak, Kuwait y Unión de Emiratos Árabes) y el $94 \%$ de las mismas controladas por tan sólo 15 países. En términos de producción sólo 5 países (Estados Unidos, Arabia Saudí, Federación REE.UU., Canadá e Irak) controlan más del 50\% de la producción mundial. Sin embargo, gran parte de los mayores consumidores mundiales o no tienen producción o si la tienen, no es en cantidad suficiente para cubrir sus necesidades debiendo requerir de importaciones.

Así, mientras unos países se configuran como exportadores netos (Arabia Saudí, Federación REE.
UU., Canadá, Irán y México), el resto de los primeros 20 consumidores a nivel mundial son importadores netos (Estados Unidos, China, Japón, Brasil, Corea del Sur y Alemania.

Con la información obtenida sobre producción y consumo de petróleo se observa que las necesidades de transporte de este producto son muy elevadas encontrando dos posibilidades logísticas para conectar los centros de producción con los de consumo: transporte mediante tuberías y transporte mediante medios marítimos.

Centrándonos en el tráfico marítimo, el movimiento de crudo de petróleo alcanzó en el año 2018 1,875 millones de toneladas cargadas y 2,034 millones de toneladas descargadas. La estimación provisional para el año 2020 alcanza los 13,500 billones de toneladasmillas transportadas de petróleo y derivados. Esto supone un $17 \%$ del tráfico marítimo mundial [51].

Bajo esta perspectiva parece razonable pensar que tanto la industria como el mundo científico deberían haber prestado un especial interés a los procesos de optimización de estos tráficos y, dentro de este campo, a la Investigación Operativa (en adelante OR), con

Tabla 1. Producción y consumo de petróleo: países TOP en 2018.

\begin{tabular}{|c|c|c|c|c|c|c|c|c|c|}
\hline & \multicolumn{3}{|c|}{ Reservas } & \multicolumn{3}{|c|}{ Producción de Petróleo } & \multicolumn{3}{|c|}{ Consumo de Petróleo } \\
\hline & País & $\begin{array}{l}\text { x } 1000 \text { Millones } \\
\text { de Toneladas }\end{array}$ & $\%$ & País & $\begin{array}{c}\text { Millones } \\
\text { Toneladas }\end{array}$ & $\%$ & País & $\begin{array}{c}\text { Millones } \\
\text { Toneladas }\end{array}$ & $\%$ \\
\hline 1 & Venezuela & 48,0 & $19,6 \%$ & EE.UU. & 669,4 & $15,0 \%$ & EE.UU. & 892,8 & $19,7 \%$ \\
\hline 2 & Arabia Saudí & 40,9 & $16,7 \%$ & Arabia Saudí & 578,3 & $12,9 \%$ & China & 628,0 & $13,9 \%$ \\
\hline 3 & Canadá & 27,1 & $11,1 \%$ & Fed. REE.UU. & 563,3 & $12,6 \%$ & India & 236,6 & $5,2 \%$ \\
\hline 4 & Irán & 21,4 & $8,8 \%$ & Canadá & 255,5 & $5,7 \%$ & Japón & 175,5 & $3,9 \%$ \\
\hline 5 & Iraq & 19,9 & $8,1 \%$ & Iraq & 226,1 & $5,1 \%$ & Arabia Saudí & 156,1 & $3,4 \%$ \\
\hline 6 & Fed. Rusa & 14,6 & $6,0 \%$ & Irán & 220,4 & $4,9 \%$ & Fed. REE.UU. & 146,3 & $3,2 \%$ \\
\hline 7 & Kuwait & 14,0 & $5,7 \%$ & China & 189,1 & $4,2 \%$ & Brasil & 141,3 & $3,1 \%$ \\
\hline 8 & EAU & 13,0 & $5,3 \%$ & EAU & 177,7 & $4,0 \%$ & Corea del Sur & 122,3 & $2,7 \%$ \\
\hline 9 & US & 7,3 & $3,0 \%$ & Kuwait & 146,8 & $3,3 \%$ & Alemania & 109,2 & $2,4 \%$ \\
\hline 10 & Libia & 6,3 & $2,6 \%$ & Brasil & 140,3 & $3,1 \%$ & Canadá & 105,2 & $2,3 \%$ \\
\hline 11 & Nigeria & 5,1 & $2,1 \%$ & México & 102,3 & $2,3 \%$ & Irán & 82,1 & $1,8 \%$ \\
\hline 12 & Kazajistán & 3,9 & $1,6 \%$ & Nigeria & 98,4 & $2,2 \%$ & Indonesia & 80,1 & $1,8 \%$ \\
\hline 13 & China & 3,5 & $1,4 \%$ & Kazajistán & 91,2 & $2,0 \%$ & México & 78,8 & $1,7 \%$ \\
\hline 14 & Qatar & 2,6 & $1,1 \%$ & Noruega & 83,1 & $1,9 \%$ & Francia & 76,4 & $1,7 \%$ \\
\hline 15 & Brasil & 2,0 & $0,8 \%$ & Qatar & 78,5 & $1,8 \%$ & Singapur & 75,2 & $1,7 \%$ \\
\hline & Resto Mundo & 14,8 & $6,1 \%$ & Resto Mundo & 853,8 & $26,9 \%$ & Resto Mundo & 1423,4 & $31,4 \%$ \\
\hline & Total Mundo & 244,1 & $100,0 \%$ & Total Mundo & 4474,3 & $100 \%$ & Total Mundo & 4529,3 & $100,0 \%$ \\
\hline
\end{tabular}

Fuente: BP Statistical Review of World Energy 2019. 
el objetivo de profundizar en el diseño de modelos de simulación dirigidos hacia la optimización de la operativa portuaria. Pero esta modalidad de tráfico no ha gozado de una popularidad académica tan sólida como en el caso de otros ámbitos de carga, como el de la mercancía contenerizada [28].

En particular, hasta el momento presente, no se ha hecho una recopilación, análisis y clasificación de los modelos de simulación que se han publicado en los últimos años sobre el tráfico marítimo de crudo de petróleo tal y como sí que ha pasado con el sector de los contenedores [23]. Cuestión que trata de suplirse con esta investigación.

\section{ESTADO DEL ARTE}

En muchas ocasiones las aplicaciones militares resultan pioneras en diversos campos científicos. Los procesos de modelización del tráfico marítimo asociado al crudo de petróleo y sus derivados, son un caso claro de esto. De este modo, Tompkins [50] desarrolla un algoritmo para optimizar el suministro de combustible a los barcos de la armada estadounidense. Sus estudios son continuados desde un punto de vista más práctico por Flood [28]. Este último autor, en base a los datos reales del año 1950 de viajes en lastre de los buques de abastecimiento de la flota, es decir, una vez que han suministrado el combustible a los barcos que alimentan, concluye que un modelo computacional de selección de rutas lograría ahorrar hasta un 5\% de las distancias recorridas.

Dantzig y Fulkerson [21] introducen por primera vez lo que se conoce como técnicas Mixed Integer Problem (en adelante, MIP) en el campo de programación de buques tanqueros; lo que se ha denominado posteriormente Inventory Routing Problem (en adelante, IRP). La influencia posterior de estos autores ha sido muy relevante en este campo.

Briskin [19] estudia como minimizar la flota de petroleros que cumplan un programa de cargas fijo (conocidas de antemano las fechas de carga y descarga). Estos investigadores reconocen que se trata de problemas de gran escala que, al abordarse con técnicas MIP, se simplifican sustancialmente. Para justificar la introducción de estas técnicas indica que la mayoría de las variables presentan limitaciones para hacerse cero y que las fórmulas de minimización son relativamente simples. Los autores presentan también un modelo para realizar una programación de descargas asociadas a barcos de granel líquido en una aplicación con varios puertos de destino y buques. Para este autor, los trabajos de Dantzig y Fulkerson [21] resolvían el problema de qué barco iba y a dónde, sin embargo, en esta investigación, también, se asumen fechas de carga/descargas predeterminadas y sin capacidad de que el modelo proporcione cuáles son esas fechas óptimas. El modelo desarrollado por Briskin permite un funcionamiento logístico en el que se producen descargas parciales (un barco descarga en diferentes destinos) y donde la flota es heterogénea.

Bellmore et al. [12] continúan avanzando con los trabajos de sus predecesores. Su innovación se centra en introducir en el modelo la posibilidad de que la flota sea insuficiente y de que alguno de los cargamentos tenga que ser cancelado (posibilidad no permitida en el problema de Dantzig y Fulkerson [22] para los que el número de buques tiene que ser siempre suficiente para tomar las cargas que están disponibles).

Bellmore et al. [13] introduce nuevas mejoras en el modelo al eliminar ciertas restricciones. Una es permitir que las cargas sean parciales (no sólo las descargas tal y como ya había introducido Briskin [19], y otra que la flota sea heterogénea. La técnica que utilizan estos autores sigue siendo MIP con introducción de algoritmos branch and bound.

Everett et al. [25] sintetizan parte de los resultados de un estudio de alcance mucho mayor encargado por la administración de Estados Unidos para planificar el desarrollo de una flota de buques de bandera propia. Este proyecto (1969) analiza el número y tamaño óptimo de tres tipos de buques: tanqueros para productos petrolíferos y crudo; tanqueros para transporte de los principales graneles sólidos y OBO's (Ore-Bulk-Oil Tanker) para atender 8 tipos de productos (crudo, mineral de hierro, carbón, fosfato, grano, bauxita, manganeso y cromo).

El objetivo de este estudio era preparar un paquete de medidas de estímulo para lograr que los astilleros norteamericanos resultasen competitivos y en base a una cartera de pedidos de gran escala pudiesen acometer unas inversiones de gran alcance en los tiempos y con el coste requerido. El programa resultó en una recomendación para construir 300 barcos en el periodo 1972-1982. 
El modelo que implementan los autores se engloba dentro de programación lineal y era resuelto por el sistema "Ophelie" de Control Data Corporation, mientras que las matrices necesarias para alimentar el modelo se construyeron con CDC's Matrix Generation Lenguage.

Mckay y Hartley [37] utilizan una formulación similar a la de autores anteriores, en este caso para optimizar el suministro de productos petrolíferos a la flota norteamericana y, por tanto, con un fin militar que recuerda a los primeros trabajos de Tompkins [50] y Flood [28] y que más tarde serían abordados en Fisher y Rosenwein[26].

Baker [8] presenta las investigaciones para la petrolera Exxon y, en concreto, su programa interactivo de buques, estudios que vienen referenciados por otros autores [6].

Daskin y Walton [22] analizan el "lightering" o proceso de transbordo de un buque de mayores dimensiones a otro más pequeño para hacer una distribución regional del producto. Estos autores explican como por razones económicas en muchos casos conviene realizar el transporte del crudo en buques de grandes dimensiones (clases VLCC o ULCC) cuando se trata de viajes transoceánicos para después transbordar la carga a otros de menores dimensiones que puedan acceder a terminales de descarga con limitaciones de calado. Con este objetivo desarrollan un modelo de colas en el que el primer servidor es el VLCC/ ULCC y sus clientes demandantes del servicio los buques más pequeños que se encuentran sin carga $y$, el segundo servidor es la terminal portuaria y sus clientes los barcos de menores dimensiones cargados desde los VLCC/ULCC.

En su modelo los autores asumen que tanto la distribución de tiempos de servicio en la operación de transbordo y descarga en puerto como la distribución de la ley de llegada de buques petroleros es exponencialmente distribuida (llegadas aleatorias) introduciendo como variante un modelo $\mathrm{M} / \mathrm{E}_{\mathrm{K}} / \mathrm{n}$ para las demoras en los buques VLCC. El modelo por tanto se resuelve como un clásico ejemplo de colas cíclicas.

Brown et al. [20] tratan la programación de cargamentos de crudo de petróleo desde Oriente Medio a Europa y Estados Unidos mediante el software Elastic Set Partitioning, modelo que sería después extendido en Bausch et al. [11]. Se trata de una aplicación práctica desarrollada para la petrolera Chevron. Según estos investigadores esta herramienta ha venido utilizándose con éxito en el sector del oil \& gas ya que permite manejar estructuras de costes no lineales (como los que se dan en estas infraestructuras) y aplicar un amplio rango de reglas procedimentales, todo ello con unos requerimientos simples capaces de ser acoplados en microordenadores.

Para estos autores, las clásicas aproximaciones lineales a modelos de transporte donde el modelo se alimenta con variables como el número de medios (camiones o buques), distancias recorridas o tiempo utilizado son únicamente válidas cuando las características de las flotas son uniformes (mismo tipo de medios con mismo tipo de coste unitario). Sin embargo, la realidad difiere de estas condiciones ideales y allí donde la flota es heterogénea aparecen efectos no lineales (diferentes estructuras de costes y costes unitarios de cada medio). El software utilizado Elastic Set Partitioning es capaz de trabajar con estos efectos no lineales siendo una aplicación de la familia MIP. Los autores describen la herramienta y algunas aplicaciones de su utilización. Su modelo tiene en cuenta todos los componentes (fijos y variables) de coste de la flota utilizada para el transporte (incluyendo los costes unitarios del buque, sus consumos, costes portuarios y tránsito en canales) y da como resultado la velocidad óptima, la mejor ruta y la programación más ajustada en tiempos computacionales muy cortos.

Bjørstad y Haugland [15] presentan un modelo estocástico para el análisis del proceso de mezclado y homogeneización (blending) de gasolinas en una refinería considerando la incertidumbre que introduce el tiempo entre llegada de buques, su orden de llegada y el tipo de buques que van a recalar a puerto. El modelo presentado es de naturaleza no lineal y tiene en consideración dos aspectos diferenciados: por un lado, la mezcla de producto que hay que realizar en cada cargamento para cumplir las condiciones de calidad requeridas para este barco, en concreto, y la distribución de los diferentes componentes producidos en la refinería para proporcionar materiales de diferentes composiciones y calidades. En síntesis, estos autores tratan de generar un modelo de transporte que tenga en consideración la cantidad y calidad del producto requerido por cada 
uno de los clientes de la refinería y, que ayude a los gestores de la instalación a programar la llegada de los buques y a realizar las operaciones en ellos.

Breton y El-Hachem [18] trabajan sobre un modelo estocástico para la planificación de slots de los barcos que llegan a una refinería para descargar crudo o retirar producto refinado en horizontes de medio y largo plazo. Para estos autores, las soluciones de tipo determinista aplicadas en el pasado sobre procesos que, como el llevado a cabo en las refinerías, tienen una naturaleza muy estocástica, han conducido a errores en cuanto a las capacidades de las instalaciones de refino, la gestión de los stocks y el suministro de crudo para realizar la producción. Así, en su artículo, discuten otros procedimientos estocásticos que mejoren el análisis de este tipo de instalaciones, resaltando los problemas a los que deben enfrentarse en su formulación, indicando como pueden superarse y presentando posibilidades de desarrollo y procedimientos computacionales concretos.

Como ejemplo, presentan una herramienta específica denominada MARKAL-Québec $[9,10,14]$ que cuenta con un módulo específico de gestión de refinerías y que es una evolución del primer modelo conocido como MARKAL que había sido desarrollado por la Agencia Internacional de la Energía como un modelo general para el sector de la energía [27].

Perakis y Bremer [42] y Bremer y Perakis [17] continúan con la línea de investigación para la empresa Chevron que había comenzado Brown et al. [20].

Shah [46] analiza técnicas de programación para el suministro de crudo de petróleo a una refinería, y Lee et al. [35] trabajan con técnicas MIP para la programación a corto plazo de buques que descargan crudo de petróleo en una refinería, introduciendo en el modelo las restricciones que supone la gestión del almacenamiento en tanques (IRP).

Andrews et al. [7] estudian el caso específico del transbordo o lightering en la bahía del río Delaware (EE.UU.). El modelo se utiliza con una finalidad estratégica para evaluar diferentes posibilidades de inversión con las que realizar la operación de la manera más eficiente (es decir, la de menor coste). El transbordo y suministro estudiado entra dentro de un caso real promovido por la empresa Maritrans (encargada de prestar dicho servicio). Los estudios para esta empresa son continuados con posterioridad en Mudrageda et al. [40], Mudrageda y Murphy [41] y Almaz y Altiok [5].

Al-Yakoob [6] aborda la optimización en el diseño y utilización de una flota de buques petroleros considerando un horizonte de planificación evolutivo (rolling).

Sherali et al. [47] estudian la logística de Kuwait Petroleum Corporation mediante formulación del tipo MIP, que resuelven con la ayuda del software CPLEX-4.0 construyendo un procedimiento de enrutamiento ad hoc que sigue las prácticas de programación de esta compañía ofreciendo resultados satisfactorios en los horizontes de programación, usualmente, contemplados por esta empresa.

Lin et al. [36] retoman estudios anteriores de simulación del lightering introduciendo en este caso alguna novedad. Formulan un modelo matemático en tiempo continuo que se resuelve con MILP (Mixed Integer Linear Programming) y que está inspirado en trabajos anteriores de programación a corto plazo de ciertos procesos químicos. Este procedimiento se basa en la programación de una secuencia de eventos que es asociada a cada buque (operación de amarre de un buque a otro, bombeo de crudo entre buques, desamarre de buques, viaje de buque a terminal en refinería, bombeo de buque a refinería, desamarre en el atraque de la terminal y viaje de retorno) y en la asociación a cada uno de estos eventos a una variable binaria (en la que el buque puede estar haciendo dicha tarea o no).

El modelo de estos autores introduce como novedad la posibilidad de bombeo desde distintos tanques y la posibilidad de proceder a transbordos parciales o en fases que junto con la naturaleza de tiempo continuo de su modelo se configura como la aportación más relevante. Los investigadores verifican sus resultados en tres casos experimentales y desde el punto de vista de las herramientas formulan el modelo en GAMS y lo resuelven con CPLEX 6.5 en una Workstation HP J-2240.

Los transbordos parciales se refieren al caso en el que el VLCC tiene que ser descargado en dos etapas por razones de calado. Primero se transborda parte de la 
carga en profundidades offshore y más tarde, cuando el calado disminuye, el buque se mueve a un amarre protegido donde se realiza el final del transbordo.

Reddy et al. $[44,45]$ analizan el problema de transbordo de material desde un VLCC a una refinería, pero incorporan ciertas novedades sobre modelos anteriores. Retoman la idea de un modelo en tiempo continuo presentado en Lin et al. [36] pero esta vez el VLCC transporta diversos materiales en diferentes bodegas y estos pueden ser bombeados a tanques al mismo tiempo. Adicionalmente, el modelo permite trasiegos de tanque a tanque reproduciendo más fielmente la operación real de muchas de estas instalaciones. El algoritmo desarrollado evita la concentración de discrepancias (consideradas como el mayor problema de estos modelos en el pasado) descomponiendo tiempos de programación más largos en periodos más cortos, utilizando funciones de relajación que proporcionan soluciones exactas intermedias. De igual modo, son los primeros autores que proporcionan una comparación entre los resultados obtenidos con un modelo en tiempo discreto y continuo.

Mendez et al. [38, 39] abordan la resolución conjunta de la programación de barcos y el blending de gasolinas. Para ello comienzan por una interesante descripción del proceso industrial desarrollado en este tipo de instalaciones que comienza en la descarga y mezcla del crudo de petróleo y que termina en la expedición de los productos finales (gasolina, diésel, queroseno, ...) y su carga en los buques. Desde el punto de vista técnico ejecutan el problema mediante técnicas MILP, que resuelven tanto con técnicas de tiempo discreto como con representaciones del modelo en tiempo continuo lo que supone una novedad sobre desarrollos anteriores.

Persson y Göthe-Lundgren [43] desarrollan un modelo para formular el problema IRP asociado a una refinería y el transporte de los productos en ella elaborados a depósitos de distribución aguas abajo de la cadena de suministro. Posteriormente, comparando su modelo con las refinerías del grupo Nynas (3 refinerías), utilizando cuatro productos en el transporte y contabilizando un total de 15 depósitos.

Huang y Karimi [31] estudian también el problema de transbordo de crudo entre buques. Los buques utilizados por estos autores permitían una compartimentación de la carga por calidades en diferentes tanques. Estos barcos sirven a diferentes refinerías dentro de una misma área geográfica. A parte de esta característica, los autores introducen nuevas funcionalidades al modelo como son la limitación del número de operaciones de transferencia que se realizan simultáneamente o la variación de la densidad del crudo con el tiempo (y con ello de los ritmos de transbordo). Desarrollan dos modelos MILP en tiempo continuo basados en programación de slots con dos funciones objetivo: por un lado, minimizar las esperas de los buques y, por otro, minimizar los costes de fletamento. Además, realizan una nueva comparación entre su modelo y los desarrollados por otros autores bajo técnicas discretas, llegando a la conclusión de que su modelo proporciona soluciones óptimas con mayor velocidad.

Kobayashi y Kubo [34] tratan la optimización de la programación de buques tanque mediante técnicas de generación de columnas y redes tiempo-espacio. Su base experimental es el problema de transporte de productos petrolíferos en Japón, que se desarrolla en un ambiente con grandes incertidumbres (eventos inesperados desde el punto de vista de las operaciones marítimo/portuarias y evolución dinámica de la demanda) que obliga a revisar los planes de transporte constantemente y, por ello, hacen aconsejable la adopción de modelos que ayuden a tomar las mejores decisiones. Desde el punto de vista computacional los autores descomponen el problema en dos sub-problemas (el primero relativo a la ruta de los barcos y, el segundo, se refiere al establecimiento de relaciones entre los puertos de carga y descarga) que resuelven mediante las técnicas de generación de columnas y redes tiempo-espacio. En los casos investigados indican que se ha conseguido un ahorro de costes entre el 5 y el $16 \%$ sobre los que se habrían obtenido sin la implementación del modelo por ellos desarrollado.

Kobayashi [33] utiliza la misma base experimental de Kobayashi y Kubo [48] para explorar desde una perspectiva más estratégica técnicas de programación dinámica que permitan trabajar con horizontes de programación a largo plazo. En su modelo la partición del problema es modificada de tal manera que pueda ser abordada desde técnicas de generación de rutas.

Furman et al. [29] analizan bajo técnicas de simulación MIP para resolver el IRP las operaciones 
marítimas de la petrolera ExxonMobil haciendo hincapié en la componente downstream de sus actividades. En concreto, estudian el suministro de gasoil desde diferentes puntos de suministro en Europa a sus refinerías en Norte América.

Engineer et al. [24] acoplan un algoritmo para tener en cuenta los precios del producto, que se ha definido como Pricing, Inventory \& Scheduling Combined Problem (en adelante PISCP) y lo aplican a una empresa petrolera. La introducción del precio en la formulación se considera un avance muy relevante en la arquitectura de este tipo de modelos que responde a las necesidades reales del mercado.

Hennig et al. [30] utilizan técnicas MIP para tratar el transporte de crudo de petróleo por vía marítima, en un problema ya clásico. Sin embargo, su objetivo es encontrar las rutas óptimas (con menor coste) teniendo en consideración una composición dada de flota (con características heterogéneas) que tiene que transportar múltiples productos. Para ello diseñan un modelo del tipo "path flow" en el que cada camino es una ruta. En su modelo introducen como elemento más llamativo las características (calidad) del material en el puerto de origen y destino que no coinciden previamente por lo que existe cierta arbitrariedad que hay que gestionar e introducir en el modelo.

Agra et al. [1, 2, 3, 4] en un contexto de optimización de rutas de buques (problemas IRP o RSP -Routing and Scheduling Problem-) analizan el transporte de corta distancia y cabotaje de fueloil en Cabo Verde. Su objetivo es determinar las políticas de enrutamiento óptimas, minimizando los costes de operación, pero manteniendo en todo momento una política de stocks dentro de límites establecidos. Desde el punto de vista computacional proponen una formulación del tipo arc-load flow. En su estudio toman horizontes de planificación de varios meses.

Siddiqui y Verma $[48,49]$ vuelven a abordar el problema de programación y optimización de rutas en el transporte de crudo de petróleo desde Oriente Medio a Europa y Norte América. Estos autores, a la clásica aproximación desde la perspectiva de optimización de coste mediante técnicas MIP, tienen en consideración una segunda componente como es el riesgo asociado a accidentes y episodios de contaminación accidental. En tal sentido hablan de una función con dos objetivos (coste y riesgo) que aplican a una flota heterogénea de petroleros. En su investigación concluyen que no siempre la ruta más corta es la óptima pues es posible que introduzca mayores riesgos y costes asociados a seguros y potenciales indemnizaciones.

Más recientemente, Wang et al. [52] en un estudio que trata de servir de base a la toma de decisiones de inversión en la red de puertos de China, analizan las rutas a seguir por los buques de petróleo que operan en el país e introducen como factor de análisis y selección no sólo el coste del transporte sino el factor del riesgo y fiabilidad considerando la incertidumbre de disponibilidad de los puertos o nodos de la red de transporte (afectados por eventos extremos). El modelo desarrollado por estos autores, también de naturaleza MIP, define una función de optimización bi-objetivo en el que en cada nodo sólo dos estados son posibles (operativo o no operativo). Como limitaciones de su modelo los propios autores indican que: i) todos los importadores se tratan como un único ente, cuando el volumen de importación de cada exportador de petróleo crudo varía y por lo tanto pudiera tratarse como una variable decisión más (lo que permitiría la simultánea optimización de la estrategia de volumen de importación y estrategia de transporte) y ii) no existe segregación de rutas por tipo de buques (aun cuando no todos las rutas pueden ser seguidas por todo tipo y tamaño de buques). Los propios autores proponen para un futuro tratar el número de viajes para tipos de embarcaciones específicos en diferentes caminos como otra variable de decisión adicional.

\section{MODELIZACIÓN DE TRÁFICOS DE CRUDO DE PETRÓLEO Y SUS DERIVADOS: CLASIFICACIÓN TEMÁTICA, EVOLUCIÓN E INNOVACIONES INTRODUCIDAS}

Como se ha constatado, el uso de modelos computacionales en el ámbito de los tráficos de crudo de petróleo y sus productos derivados cuenta con un recorrido histórico de al menos 65 años. En todo este periodo se han venido configurando diversas líneas de investigación en función del problema logístico concreto que trataban de abordar.

De igual modo, con el tiempo se han venido eliminando determinadas restricciones de los 
modelos iniciales e incorporándose innovaciones que han hecho evolucionar los modelos hacia las necesidades reales de la industria y las características específicas de los entornos de operaciones portuarias.

Si la atención se centra en el primer atributo, se puede indicar que tres son los grandes grupos en los que podríamos dividir estos estudios.

Bunker Optimization Problem (en adelante BOP). Estos modelos trataban de optimizar las rutas de buques que suministran bunker a una flota amplia de embarcaciones. Son los modelos más antiguos, desarrollados con fines militares y con el objetivo de optimizar el suministro de combustible a barcos de la armada. Dentro de este grupo destacan Tommkins[50], Floord [28], Mckay y Hartley [37] y Fisher y Rosenwein [27]. El BOP evolucionó a una variante del mismo con fines civiles. Se trata de la optimización de operaciones de transbordo de crudo de petróleo desde grandes buques petroleros a barcos más pequeños. Los primeros encargados de realizar las tareas de transporte desde grandes centros de producción o carga hasta áreas cercanas a refinerías situadas en puertos con problemas de calado y los segundos encargados de hacer llegar el material a los propios muelles de las refinerías o de los oleoductos que las alimentan, denominado Lightering Operation Problem (en adelante LOP), y lo han abordado Daskin y Walton [22], Andrews et al. [7], Lin et al. [36], Mudrageda et al. [39], Reddy et al. [44, 45], Huang y Karimi [32], Mudrageda et al. [40] y Almaz y Altiok [5].

Inventory and Routing Problem (IRP). Es un problema clásico de OR que trata de optimizar la ruta de buques que recalan en determinados puertos realizando la programación de slots de carga/descarga considerando el nivel de inventario del producto almacenado en los puertos y la capacidad de almacenamiento en los mismos. En el caso concreto de productos petrolíferos este problema se ha tratado por Dantzig y Fulkerson [21], Briskin [19], Bellmore et al. [12, 13], Everett et al. [25], Baker [8], Brown et al. [20], Perakis y Bremer [42], Bremer y Perakis [17], Shah [46], Lee et al. [35], Al-Yakoob [6], Sherali et al. [47], Persson y Göthe-Lundgren [43], Kobayashi y Kubo [34], Kobayashi [33], Furman et al. [29], Hennig et al. [30], Agra et al. [1, 2, 3, 4] y Siddiqui y Verma [48, 49].
Cargo Scheduling with Blending Problem (CSBP). Bajo este epígrafe genérico se consideran los trabajos de investigación que tratan de optimizar las programaciones de atraques de buques en una terminal por criterios volumétricos, por la propia calidad de los productos básicos entregados así como por las características del proceso industrial necesario para realizar el mezclado de los mismos para dar un material que cumpla las especificaciones de determinados clientes. A esta categoría pertenecen los trabajos de Bjørstad y Haugland [15], Breton y El-Hachem [18] y Mendez et al. [38, 39].

Las sucesivas mejoras materiales y de infraestructura han ido introduciendo, asimismo, intensas innovaciones en los modelos de simulación constituyéndose, debido a su naturaleza, en una categorización adicional. Se trata, fundamentalmente de: la introducción de una flota heterogénea con buques de diferentes tamaños y en la que las fechas de carga y descarga no son fijas [19]; la consideración de cargas y descargas parciales $[19,13]$; la introducción de restricciones en cuanto al número de buques disponibles, lo que permitiría cancelar cargamentos [12]; la posibilidad de que el operador interactúe con el modelo realizando una programación más dinámica [8]; la posibilidad de introducir en el proceso de optimización económica costes no lineales [20]; la introducción de criterios de calidad en el producto [15]; la consideración conjunta en el mismo modelo de la descarga de crudo y de la carga de refinados en un sistema de gestión integral de la terminal [18]; la introducción de horizontes evolutivos de programación [6, 47]; la introducción de operaciones en sucesivas fases y en ambientes continuos [36]; la posibilidad de bombeo simultáneo desde diferentes tanques de un mismo buque $[44,45]$; las mejoras en cuanto al horizonte de simulación, permitiendo programaciones a más largo plazo [44, 45, 33, 1, 2, 3, 4]; la posibilidad de ir variando las características del producto a lo largo de la operación y con ello modificando los rendimientos de la misma [32]; la introducción de una variante en la que en el enrutamiento óptimo también se considera el precio del producto [24]; la consideración de compatibilidad entre puertos de carga y descarga [30]; y, por último, la introducción, como función objetivo, del riesgo asociado a cada una de las rutas que permite una optimización adicional de las mismas [48, 49].

En la Tabla (2) se presentan los autores con indicación del problema que resuelven y la técnica utilizada 
Tabla 2. Innovaciones y principales restricciones de los modelos aplicados en el tráfico de productos petrolíferos y sus derivados

\begin{tabular}{|c|c|c|c|c|}
\hline Autor & Problema & Técnica & Principal innovación presentada & Principales restricciones \\
\hline Tompkins [50] & BOP & MIP & $\begin{array}{l}\text { Introducción de simulación en el campo de } \\
\text { operaciones buques petroleros. }\end{array}$ & $\begin{array}{l}\text { Desarrollo teórico sin contraste con datos } \\
\text { reales. }\end{array}$ \\
\hline Flood [28] & BOP & MIP & $\begin{array}{l}\text { Presentación de resultados prácticos en base } \\
\text { a datos reales. }\end{array}$ & Tamaño del Modelo y Rapidez de resolución. \\
\hline Dantzig y Fulkerson [21] & IRP & MIP & Introducción de simulación en el campo civil. & $\begin{array}{l}\text { Programa de Cargas y Descargas Fijo. } \\
\text { Flota homogénea. } \\
\text { NO permite cargas / descargas parciales. }\end{array}$ \\
\hline Briskin [19] & IRP & MIP & $\begin{array}{l}\text { Flota heterogénea. } \\
\text { Fecha de carga y descarga no prefijadas. } \\
\text { Descargas Parciales. }\end{array}$ & $\begin{array}{l}\text { Flota ilimitada (todas las cargas pueden } \\
\text { atenderse y no se cancelan. } \\
\text { No permite cargas parciales. }\end{array}$ \\
\hline Bellmore et al. [12] & IRP & MIP & $\begin{array}{l}\text { Flota limitada (Posibilidad de cancelación } \\
\text { de cargas). }\end{array}$ & Único producto. \\
\hline Bellmore et al. [13] & IRP & $\begin{array}{l}\text { MIP (branch } \\
\text { and bound) }\end{array}$ & Permite descargas y cargas Parciales. & Único producto. \\
\hline Everett et al. [25] & IRP & MIP(Ophelie) & $\begin{array}{l}\text { Varios tipos de productos (no sólo productos } \\
\text { petrolíferos). } \\
\text { Diferentes tipos de buques (no solo buques } \\
\text { petroleros). }\end{array}$ & $\begin{array}{l}\text { Misma calidad de producto dentro del } \\
\text { sistema grupo. }\end{array}$ \\
\hline Mckay y Hartley [37] & BOP & MIP & $\begin{array}{l}\text { Evolución del modelo de Flood (1954) } \\
\text { superando limitaciones tamaño y rapidez } \\
\text { modelo. }\end{array}$ & $\begin{array}{l}\text { Único producto. } \\
\text { = Producto de características constantes } \\
\text { durante bombeo. }\end{array}$ \\
\hline Baker [8] & IRP & MIP & Programación interactiva. & $\begin{array}{l}\text { Limitaciones Computacionales / Tamaño del } \\
\text { Modelo y Rapidez de resolución. }\end{array}$ \\
\hline Daskin y Walton [22] & LOP & MIP & $\begin{array}{l}\text { Introducción de sistema cíclicos con varias } \\
\text { colas en el campo de terminales/flota petróleo. }\end{array}$ & $\begin{array}{l}\text { No compartimentación en diferentes tanques. } \\
\text { Producto de características constantes durante } \\
\text { bombeo. } \\
\text { No permite operación en fases. }\end{array}$ \\
\hline Brown et al. [20] & IRP & $\begin{array}{c}\text { MIP } \\
\text { (Elastic Set } \\
\text { Partitioning) } \\
\end{array}$ & $\begin{array}{l}\text { Optimización basada en estructura de costes } \\
\text { no lineales. }\end{array}$ & Único producto. \\
\hline Bjørstad y Haugland [15] & CSBP & MIP & $\begin{array}{l}\text { Proceso estocástico de llegada de buques } \\
\text { (periodicidad, orden de llegada y tipo de } \\
\text { buque). } \\
\text { Consideración de cantidad y calidad de } \\
\text { producto. }\end{array}$ & Única Instalación / Refinería. \\
\hline Breton y El-Hachem [18] & CSBP & $\begin{array}{c}\text { MIP } \\
\text { (MARKAL- } \\
\text { Québec) }\end{array}$ & $\begin{array}{l}\text { Modelo extrapolable a diferentes terminales. } \\
\text { Tratamiento conjunto de descarga de crudo } \\
\text { y carga de refinado. }\end{array}$ & Única Instalación / Refinería. \\
\hline $\begin{array}{l}\text { Perakis y Bremer [42] y } \\
\text { Bremer y Perakis [17] }\end{array}$ & IRP & MIP & $\begin{array}{l}\text { Mejora en la eficiencia del modelo de Brown } \\
\text { et al. (1987). }\end{array}$ & Único producto. \\
\hline Shah [46] & IRP & MIP & $\begin{array}{l}\text { Mejora en las limitaciones (tamaño y rapidez } \\
\text { de resolución). }\end{array}$ & Consideración de un único producto (crudo). \\
\hline Lee et al. [35] & IRP & MIP & $\begin{array}{l}\text { Mejoras en la consideración de restricciones } \\
\text { en gestión de almacenamiento. }\end{array}$ & Plazos de programación a corto plazo. \\
\hline
\end{tabular}

junto con las principales restricciones de su modelo y las innovaciones propuestas. Desde el punto de vista computacional en todos los casos analizados la técnica utilizada ha sido Mixed Integer Problem (MIP) con la introducción de diferentes algoritmos a lo largo de los años.

\section{CONCLUSIONES}

La modelización del tráfico de crudo de petróleo y sus derivados está poco presente en la literatura científica si la comparamos con la aplicada a otros negocios como el de los contenedores. Esta falta 


\begin{tabular}{|c|c|c|c|c|}
\hline Autor & Problema & Técnica & Principal innovación presentada & Principales restricciones \\
\hline $\begin{array}{l}\text { Andrews et al. [7] } \\
\text { Mudrageda et al. [40] } \\
\text { Mudrageda y Murphy [41] y } \\
\text { Almaz y Altiok [5] }\end{array}$ & LOP & MIP & $\begin{array}{l}\text { Enfoque estratégico (análisis de inversiones } \\
\text { necesarias para optimización de la operación). }\end{array}$ & $\begin{array}{l}\text { Buques no compartimentados en tanques. } \\
\text { Producto de características constantes } \\
\text { durante bombeo. } \\
\text { No permite operación en fases. }\end{array}$ \\
\hline $\begin{array}{l}\text { Al-Yakoob [6] } \\
\text { Sherali et al. [47] }\end{array}$ & IRP & $\begin{array}{c}\text { MIP } \\
\text { (CPLEX-4.0) }\end{array}$ & $\begin{array}{l}\text { Introducción de horizontes evolutivos de } \\
\text { programación. }\end{array}$ & $\begin{array}{l}\text { Limitaciones en cuanto al tamaño del modelo } \\
\text { y su tiempo de resolución. }\end{array}$ \\
\hline Lin et al. [36] & LOP & $\begin{array}{c}\text { MIP } \\
(\text { GAMS y } \\
\text { CPLEX 6.5) }\end{array}$ & $\begin{array}{l}\text { Introducción de modelos en tiempo continuo } \\
\text { dentro del LOP. } \\
\text { Posibilidad de operación en fases (transbordos } \\
\text { parciales). }\end{array}$ & $\begin{array}{l}\text { Horizontes de programación de corto plazo. } \\
\text { Producto de características constantes } \\
\text { durante bombeo. }\end{array}$ \\
\hline Reddy et al. $[44,45]$ & LOP & MIP & $\begin{array}{l}\text { Comparación de resultados con modelos } \\
\text { de tiempo continuo y eventos discretos. } \\
\text { Posibilidad de bombeo simultáneos desde } \\
\text { distintos tanques. } \\
\text { Horizontes de programación de largo } \\
\text { plazo (técnicas de relajación encontrando } \\
\text { soluciones parciales en tiempos más cortos). }\end{array}$ & $\begin{array}{l}\text { Producto sin variación de características } \\
\text { durante el bombeo (densidad constante). }\end{array}$ \\
\hline Mendez et al. $[38,39]$ & CSBP & MILP & $\begin{array}{l}\text { Introducción de modelo en tiempo continuo } \\
\text { al problema de CSBP (comparación con } \\
\text { modelos DES). }\end{array}$ & Única Instalación / Refinería. \\
\hline Persson y Göthe-Lundgren [43] & IRP & MIP & $\begin{array}{l}\text { Gestión activa de varios productos e } \\
\text { instalaciones simultáneamente. }\end{array}$ & \\
\hline Huang y Karimi [31] & LOP & MILP & $\begin{array}{l}\text { Posibilidad de limitar número de operaciones } \\
\text { simultáneas. } \\
\text { Posibilidad de variar la densidad del producto } \\
\text { durante la evolución. } \\
\text { Combinación de dos funciones objetivos } \\
\text { (minimización coste flete y tiempos de espera). }\end{array}$ & No consideración de operaciones en fases. \\
\hline Kobayashi y Kubo [34] & IRP & MIP & $\begin{array}{l}\text { Técnicas de descomposición, generación de } \\
\text { columnas y redes tiempo-espacio. } \\
\text { Descomposición en subproblemas (enruta- } \\
\text { miento óptimo y conexión de puertos de } \\
\text { carga y descarga) }\end{array}$ & Horizonte de corto plazo. \\
\hline Kobayashi [33] & IRP & MIP & $\begin{array}{l}\text { Mejora del modelo de Kobayashi y Kubo } \\
\text { (2010) con técnicas dinámicas que permite } \\
\text { horizontes de largo plazo. }\end{array}$ & $\begin{array}{l}\text { No consideración de compatibilidad de } \\
\text { productos en diferentes puertos. }\end{array}$ \\
\hline Furman et al. [29] & IRP & MIP & $\begin{array}{l}\text { Introducción del foco en actividades } \\
\text { downstream (suministro de gasoil) con } \\
\text { modelo de mayor tamaño. }\end{array}$ & $\begin{array}{l}\text { No consideración de compatibilidad de } \\
\text { productos en diferentes puertos. }\end{array}$ \\
\hline Engineer et al. [24] & IRP & MIP & $\begin{array}{l}\text { Introducción del precio del producto en } \\
\text { el modelo dentro del campo de productos } \\
\text { petrolíferos (variante PISCP). }\end{array}$ & \\
\hline Hennig et al. [30] & IRP & $\begin{array}{c}\text { MIP } \\
\text { (técnicas } \\
\text { path-flow) }\end{array}$ & $\begin{array}{l}\text { Consideración de compatibilidad entre } \\
\text { puertos de carga y descarga (arbitrariedad } \\
\text { especificaciones de material en distintas } \\
\text { terminales). }\end{array}$ & \\
\hline Agra et al. $[1,2,3,4]$ & IRP & \begin{tabular}{|c|} 
MIP \\
(técnicas \\
arc-load-flow) \\
\end{tabular} & $\begin{array}{l}\text { Límites mínimos de stocks. } \\
\text { Mejora en horizontes de programación largos. }\end{array}$ & \\
\hline Siddiqui y Verma [ 48, 49] & IRP & MIP & $\begin{array}{l}\text { Introducción del riesgo en los procesos de } \\
\text { enrutamiento. }\end{array}$ & \\
\hline Wang et al. [52] & IRP & Modelo & $\begin{array}{l}\text { Introducción del concepto de riesgo y } \\
\text { fiabilidad en la disponibilidad de puerto como } \\
\text { variable adicional al coste del transporte. }\end{array}$ & $\begin{array}{l}\text { Consideran a todos los importadores como } \\
\text { un único "ente". } \\
\text { No introduce restricciones en cuanto al uso } \\
\text { de rutas por cierto tipo de buques. }\end{array}$ \\
\hline
\end{tabular}

Fuente: Elaboración propia. 
de cobertura contrasta con la importancia que este tráfico tiene en el contexto internacional del transporte marítimo (17\% de los movimientos totales en el año 2018) y el impacto en la economía global (principal fuente de generación de energía primaria en el mundo con un 32\% del total en 2018).

Desde las primeras aproximaciones académicas, procedentes del ámbito militar, los trabajos que han sido publicados se han basado mayoritariamente en la aplicación de técnicas de programación entera. Con criterio general, los estudios se pueden englobar en tres grandes grupos: el primero, se refiere a la optimización de rutas de petroleros en lo que ha venido en denominarse Inventory Routing Problem (IRP) o Routing and Scheduling Problem (RSP); el segundo tiene que ver con las operaciones de transbordo de crudo desde barcos grandes (que realizan el transporte a largas distancias desde los centros de producción a los de consumo) a barcos más pequeños (que se encargan del transporte hasta las refinerías en ubicaciones con limitaciones de calado). Esta aproximación se denomina Lightering Optimization Problem (LOP) y tiene como origen los modelos militares para resolver la optimización del proceso de suministro de bunker a la armada norteamericana Bunkering Optimization Problem (BOP); el tercer grupo también es una variante del primero, e incluye la propia gestión del tráfico de las refinerías, incorporando en los procedimientos de programación de slots, el proceso de blending de las gasolinas, e incidiendo tanto en aspectos de cantidad como de calidad del producto a ser cargado en los barcos.

Como conclusiones más significativas cabe señalar, en primer lugar, la reducción del tiempo de computación y tamaño del modelo. Esto ha venido dado por permitir la incorporación de más de un puerto de carga o descarga, operaciones conjuntas de una flota heterogénea con buques de distintos tamaños y características, así como por la modelización de más de un producto o por la extensión de los horizontes de programación a un plazo más largo.

En segundo lugar, destaca la incorporación de una mayor flexibilidad de los modelos, que se ha traducido a su vez, en la introducción de nuevas variables en los mismos (operaciones en fases, bombeos secuenciales o simultáneos, estructura de costes no lineales, variación de calidades dentro de un mismo producto y variación de las características del propio producto a lo largo de la operación).

Por último, la consideración de otras funciones objetivo (como puede ser la componente del riesgo asociado a cada una de las rutas) o estrategias de tipo comercial (incorporación del precio como un elemento más de determinación del enrutamiento óptimo).

En base a lo expuesto emergen, claramente, dos líneas de investigación futuras, que pueden resultar relevantes. Una primera profundizando en los estudios preliminares de Engineer et al. [24] en lo que se refiere a considerar en el proceso de programación no sólo la cantidad sino el precio del crudo y productos derivados. Una segunda en la que se incorporen en el análisis ciertos riesgos inherentes al tráfico marítimo de este producto $\mathrm{y}$, se investiguen alternativas de enrutamiento ante fenómenos de disrupción de las actuales cadenas logísticas por eventos inesperados tales como guerras, bloqueos comerciales a países productores, catástrofes naturales y accidentes de buques petroleros. Este tipo de estudios encuentran un primer germen en Siddiqui y Verma [48, 49].

\section{REFERENCIAS}

[1] A. Agra, M. Christiansen and A. Delgado. "Mixed integer formulations for a short sea fuel oil distribution problem". Transportation Science. Vol. 47 No 1, pp. 108-124. 2012.

[2] A. Agra, H. Andersson, M. Christiansen and L. Wolsey. "A maritime inventory routing problem: Discrete time formulations and valid inequalities". Networks. Vol. $62 \mathrm{~N}^{\circ} 4$, pp. 297-314. 2013.

[3] A. Agra, M. Christiansen, A. Delgado and L. Simonetti. "Hybrid heuristics for a short sea inventory routing problem". European Journal of Operational Research. Vol. 263 $\mathrm{N}^{\circ} 3$, pp. 924-935. 2014.

[4] A. Agra, M. Christiansen and A. Delgado. "Discrete time and continuous time formulations for a short sea inventory routing problem". Optimization and Engineering. Vol. $18 \mathrm{~N}^{\circ}$ 1, pp. 269-297. 2016.

[5] O. Almaz and T. Altiok. "Simulation modelling of the vessel traffic in Delaware river: Impact of deepening on port performance". Simulation Modelling Practice and Theory. Vol. 22, pp. 146-165. 2012. 
[6] S.M.Al-Yakoob. "Mixed-integer mathematical programming optimization models and algorithms for an oil tanker routing and scheduling problem". PhD Thesis. Virginia Polytechnic Institute and State University. 1997. Virginia, EE.UU.

[7] S. Andrews, F.H. Murphy, X.P. Wang, and S. Welch. "Modeling crude oil lightering in Delaware bay". Interfaces. Vol. $26 \mathrm{~N}^{\circ} 6$, pp. 68-78. 1996.

[8] T.E. Baker. "Interactive vessel scheduling at Exxon". CORS/TIMS/ORSA Joint National Meeting, pp. 3-6. May, 1981. Toronto, Canada.

[9] D. Baillard, C. Berger, C. Haurie and R. Loulou. "MARKAL-QUÉBEC: A report on the model and data base improvements". Groupe d'études et de recherche en analyse decisions (GERAD) Discussion paper: G-8523. 1985.

[10] D. Baillard, C. Berger, A. Haurie and R. Loulou. "Modelling the long range evolution of the oil refining industry in Canada: The MARKAL model approach". Groupe d'études et de recherche en analyse des decisions (GERAD) Discussion paper: G-87-04. 1987.

[11] D.O. Bausch, G.G. Brown and D. Ronen. "Elastic Set partitioning - A powerful tool for scheduling transportation of oil and gas". Advances in Operations Research in the Oil and Gas Industry, pp. 13-14. June 1991. Montreal, Canada.

[12] M. Bellmore, G. Bennington and S. Lubore. "A maximum utility solution to a vehicle constrained tanker scheduling problem". The Mitre Corporation. Report MRT-319. 1968.

[13] M. Bellmore, G. Bennington and S. Lubore. "A multi-vehicle tanker scheduling problem". Transportation Science. Vol. 5 $\mathrm{N}^{\mathrm{o}}$ 1, pp. 36-47. 1971.

[14] C. Berger. La modélisation des raffineries dans MARKAL-Québec. Groupe d'études et de recherche en analyse des decisions (GERAD) Discussion paper: G-85-26. 1985.

[15] H. Bjorstad and D.Haugland. "A stochastic model for gasoline blending". Proceedings of the Advances in Operations Research in the Oil and Gas Industry. 13-14 June 1991. Montreal, Canada.

[16] BP. "BP statistical review of world energy". BP p.l.c. London,UK. 2019.
[17] W.M. Bremer and A.N. Perakis. "An operational tanker scheduling optimization system: model implementation, results and possible extensions". Maritime Policy \& Management. Vol. $19 \mathrm{~N}^{\circ}$ 3, pp. 189-199.1992.

[18] M. Breton and S. El-Hachem. "Application of stochastic programming to medium- and long-term planning in the refining industry". Advances in Operations Research in the Oil and Gas Industry. 1991. Montreal, Canada.

[19] L.E. Briskin. "Selecting delivery dates in the tanker scheduling problem". Management Science. Vol. 12 N $^{\circ}$ 6, pp. 224-233.1966.

[20] G.G. Brown, G.W. Graves and D. Ronen. "Scheduling ocean transportation of crude oil". Management Science. Vol. $33 \mathrm{~N}^{\mathrm{o}} 3$, pp. 335-346. 1987.

[21] G.B. Dantzig and D.R. Fulkerson. "Minimizing the number of tankers to meet a fixed schedule". Naval Research Logistics Quarterly. Vol. 1 N $^{\circ}$ 3, pp. 217-222. 1954.

[22] M. Daskin and C.M. Walton. "An approximate analytic model of supertanker lightering operations". Transportation Research Part B Methodological. Vol. $17 \mathrm{~N}^{\mathrm{o}} 3$, pp. 201-219.1983

[23] B. Dragovic, E. Tzannatos and N. Park. "Simulation modelling in ports and container terminals: literature overview and analysis by research field, application area and tool". Flexim Service Manufacturing Journal. Vol. $29 \mathrm{~N}^{\circ}$ 1, pp. 4-34. 2016.

[24] F. Engineer, K. Furman, G. Nemhauser, M. Savelsbergh and J. Song. "A branch price-and-cut algorithm for single-product maritime inventory routing". Operations Research. Vol. 60 No 1, pp. 106-122. 2012.

[25] J. Everett, A. Hax, V. Lewinson and D. Nutts. "Optimization of a fleet of large tankers and bulkers: A linear programming approach”. Marine Technology. Vol. $9 \mathrm{~N}^{\circ} 4$, pp. 430-438.1972.

[26] M. Fisher and M. Rosenwein. "An interactive optimization system for bulk-cargo ship scheduling". Naval Research Logistics. Vol. $36 \mathrm{~N}^{\circ}$ 1, pp. 27-42. 1989.

[27] L. Fishbone and H. Abilock. "Markal, a linear-programming model for energy systems analysis: Technical description of the bnl version”. International Journal of Energy Research. Vol. 5 No 4, pp. 353-375. 1981. 
[28] M. Flood. "Application of transportation theory to scheduling a military tanker fleet". Operations Research. Vol. $2 \mathrm{~N}^{\circ}$ 1, pp. 150162. 1954.

[29] K. Furman, J. Song, G. Kocis, M. Mcdonald and P. Warrick. "Feedstock routing in the ExxonMobil downstream sector". Interfaces. Vol. 41 N$^{\circ}$ 2, pp. 149-163. 2011.

[30] F. Hennig, B. Nygreen, M. Christiansen and P. Warrick. "Maritime crude oil transportation - A split pickup and split delivery problem". European Journal of Operational Research. Vol. $218 \mathrm{~N}^{\mathrm{o}}$ 3, pp. 764-774. 2012.

[31] C. Huang and I. Karimi. "Scheduling tanker-lightering operations in crude oil transportation". Industrial \& Engineering Chemistry Research. Vol. $45 N^{\circ} 24$, pp. 80638082. 2006a.

[32] C.Huang and I. Karimi. "Scheduling ransshipment operations in maritime chemical" Transportation. Industrial \& Engineering Chemistry Research. Vol. 45 N$^{\circ}$ 6, pp. 19551973. 2006b.

[33] K. Kobayashi. "A Linear approximation of the value function of an approximate dynamic programming Approach for the Ship Scheduling Problem". Fourth International Conference on Learning and Intelligent Optimization, pp. 184-187. 2010. Venice, Italy.

[34] K. Kobayashi and M. Kubo. "Optimization of oil tanker schedules by decomposition, column generation, and time-space network techniques". Japan Journal of Industrial and Applied Mathematics. Vol. $27 \mathrm{~N}^{\mathrm{o}}$ 1, pp. 161173. 2010.

[35] H. Lee, J. Pinto, I. Grossmann and S. Park. "Mixed-integer linear programming model for refinery short-term scheduling of crude oil un-loading with inventory management". Industrial \& Engineering Chemestry Research. Vol. 35 N$^{\circ}$ 5, pp. 16301641. 1996.

[36] X. Lin, E. Chajakis and A. Christodoulos. "Scheduling of tanker lightering via a novel continuous-time optimization framework". Industrial \& Engineering Chemistry Research. Vol. $42 \mathrm{~N}^{\circ}$ 20, pp. 4441-4451. 2003.

[37] M. Mckay and H. Hartley. "Computerized scheduling of seagoing tankers". Naval Research Logistics Quarterly. Vol. $21 \mathrm{~N}^{\circ} 2$, pp. 255-264. 1974.
[38] C. Mendez, I. Grossmann, I. Harjunkoski and P. Kaboré. "Optimization techniques for blending and scheduling of oil-refinery operations". Carnegie Mellon University, Pittsburgh. 2004.

[39] C. Mendez, I. Grossmann, I. Harjunkoski and P. Kaboré. "A simultaneous optimization approach for off-line blending and scheduling of oil-refinery operations". Computers \& Chemical Engineering. Vol. $30 \mathrm{~N}^{\circ}$ 4, pp. 614-634. 2006.

[40] M. Mudrageda, F. Murphy and S. Welch. "Developing strategies for maritrans". Business Units Interfaces. Vol. 34 No 2, pp. 149-161. 2004.

[41] M. Mudrageda and F. Murphy. "An economic equilibrium model of the market for marine transportation services in petroleum products". Operations Research. Vol. 56 NN$^{\circ} 2$, pp. 278285. 2008.

[42] A. Perakis and W. Bremer. "An operational tanker scheduling optimization system: Background, current practice and model formulation". Maritime Policy \& Management. Vol. $19 \mathrm{~N}^{\circ}$ 3, pp. 177-187. 1992.

[43] J.A. Persson and M. Göthe-Lundgren. "Shipment planning at oil refineries using column generation and valid inequalities". European Journal of Operational Research. Vol. $163 \mathrm{~N}^{\circ}$ 3, pp. 631-652. 2005.

[44] P. Reddy, I.A. Karimi and R. Srinivasan. "A novel solution approach for optimizing crude oil operations". Journal of the American Institute of Chemical Engineers. Vol. 50 No 6, pp. 1177-1197. 2004.

[45] P.C.P. Reddy, I.A. Karimiand and R. Srinivasan. "A new continuous-time formulation for scheduling crude oil operations". Chemical Engineering Science. Vol. 59 N $^{\circ}$ 6, pp. 13251341. 2004.

[46] N. Shah. "Mathematical programming techniques for crude oil scheduling". Computers \& Chemical Engineering. Vol. 20 $\mathrm{N}^{\mathrm{o}}$ 2, pp. 1227-1232. 1996.

[47] H.D. Sherali, S. Al-Yakoob and M. Hassan. "Fleet management models and algorithms for an oil-tanker routing and scheduling problem". IIE transactions. Vol. $31 \mathrm{~N}^{\circ} 5$, pp. 395-406. 1999.

[48] A.W. Siddiqui and M. Verma. "An expected consequence approach to route choice in the maritime transportation of crude oil". Risk Analysis. Vol. 33 N $^{\circ} 11$, pp. 2041-2055. 2013. 
[49] A.W. Siddiqui and M. Verma. "A bi-objective approach to routing and scheduling maritime transportation of crude oil". Transportation Research Part D Transport and Environment. Vol. $37 \mathrm{~N}^{\circ}$ 1, pp. 65-78. 2015.

[50] C. Tompkins. "Discrete problems and computers". INA Conference S3-5. 17 de noviembre de 1952.
[51] UNCTAD. "Review of Maritime Transport 2019”. United Nations (UNCTAD). 2019. Geneva Switzerland.

[52] S. Wang, D. Yang and J. Lu. "A connectivity reliability-cost approach for path selection in the maritime transportation of China's crude oil imports". Maritime Policy \& Management. Vol. $45 \mathrm{~N}^{\circ}$ 5. 2018 